\title{
Neuron Conduction Induction
}

National Cancer Institute

\section{Source}

National Cancer Institute. Neuron Conduction Induction. NCI Thesaurus. Code C40924.

Neuron Conduction Induction involves initiation of activities that propagate the transfer of the electrochemical action potential process along nerve fibers away from the cell body. 